\title{
Recanalization of a distorted duodenal metal stent using a fine-gauge electrocautery
} dilator

Duodenal obstruction sometimes occurs due to pancreaticobiliary carcinoma. Because the prognosis of the advanced stage is limited, duodenal metal stents are often deployed as conservative treatment [1-4]. However, if a transpapillary bile duct stent has previously been deployed, duodenal stents can become distorted by the bile duct stent. We herein describe technical tips for recanalization of a distorted duodenal metal stent using a novel fine-gauge electrocautery dilator (Medico's Hirata Inc., Osaka Japan) [5]. This device can reduce adverse events due to burning effects compared with a conventional electrocautery dilator.

A 77-year-old man with a 4-month history of pancreatic head cancer was admitted with a duodenal obstruction, which was treated by biliary stenting using a fully covered self-expandable metal stent (FCSEMS). He experienced vomiting despite resolution of the obstructive jaundice and chemotherapy. Therefore, duodenal metal stent deployment was attempted. First, an endoscope was inserted into the duodenum, and a guidewire was advanced into the intestine across the stricture site under fluoroscopic guidance ( $\triangleright$ Fig. 1). An uncovered duodenal metal stent (HANARO stent; M.I. Tech Co., Ltd., Seoul, Korea) was then deployed (> Fig. 2). However, the vomiting persisted. Fluoroscopic imaging revealed that the duodenal stent was distorted, so an endoscope was advanced into it. The distortion was caused by the stent being placed through the mesh of the biliary stent ( $\triangleright$ Fig. 3 ). We broke the mesh of the duodenal and bile duct stents using a fine-gauge electrocautery dilator to prevent mucosal injury ( $>$ Fig.4). Fluoroscopy showed good flow of contrast medium ( $\triangleright$ Fig.5), and the vomiting immediately resolved ( Video $\mathbf{1}$ ).

In conclusion, the novel fine-gauge electrocautery dilator helped to resolve the cause of stent distortion while preventing mucosal damage.

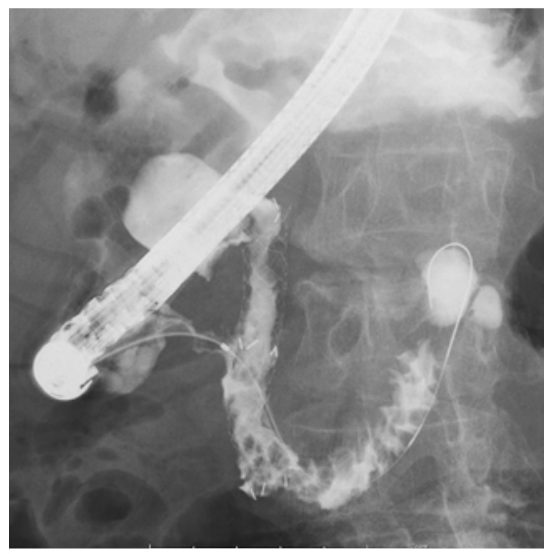

Fig. 1 The guidewire was advanced into the duodenum.

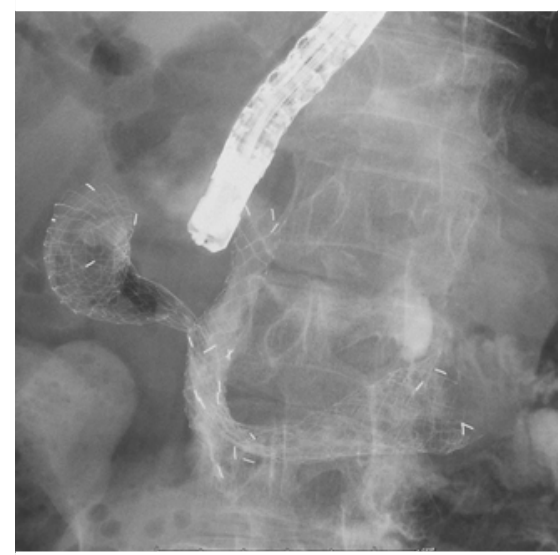

Fig. 2 A metal stent was deployed in the duodenum.

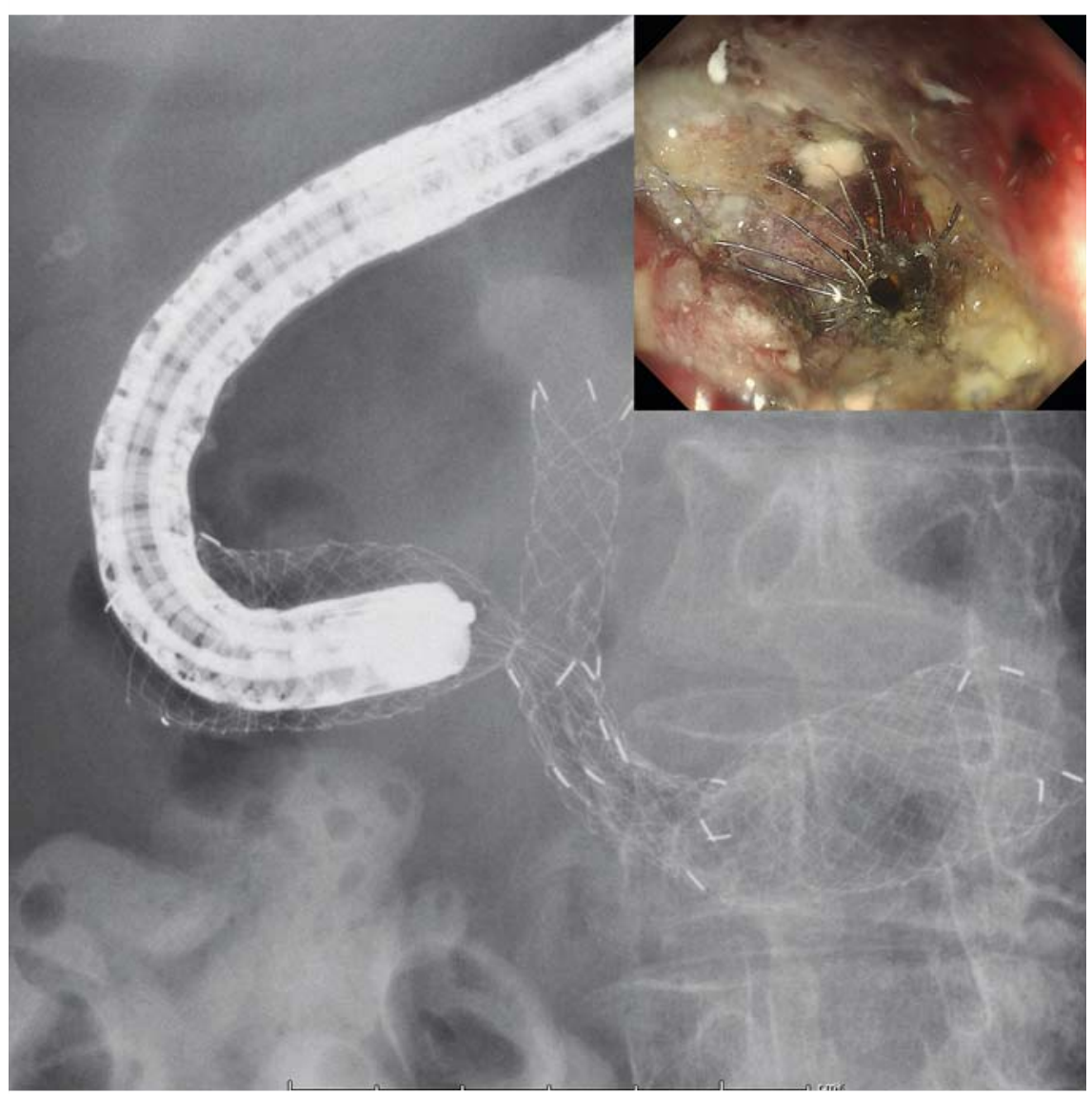

- Fig. 3 The duodenal stent had become distorted due to being positioned through the mesh of the biliary stent. 


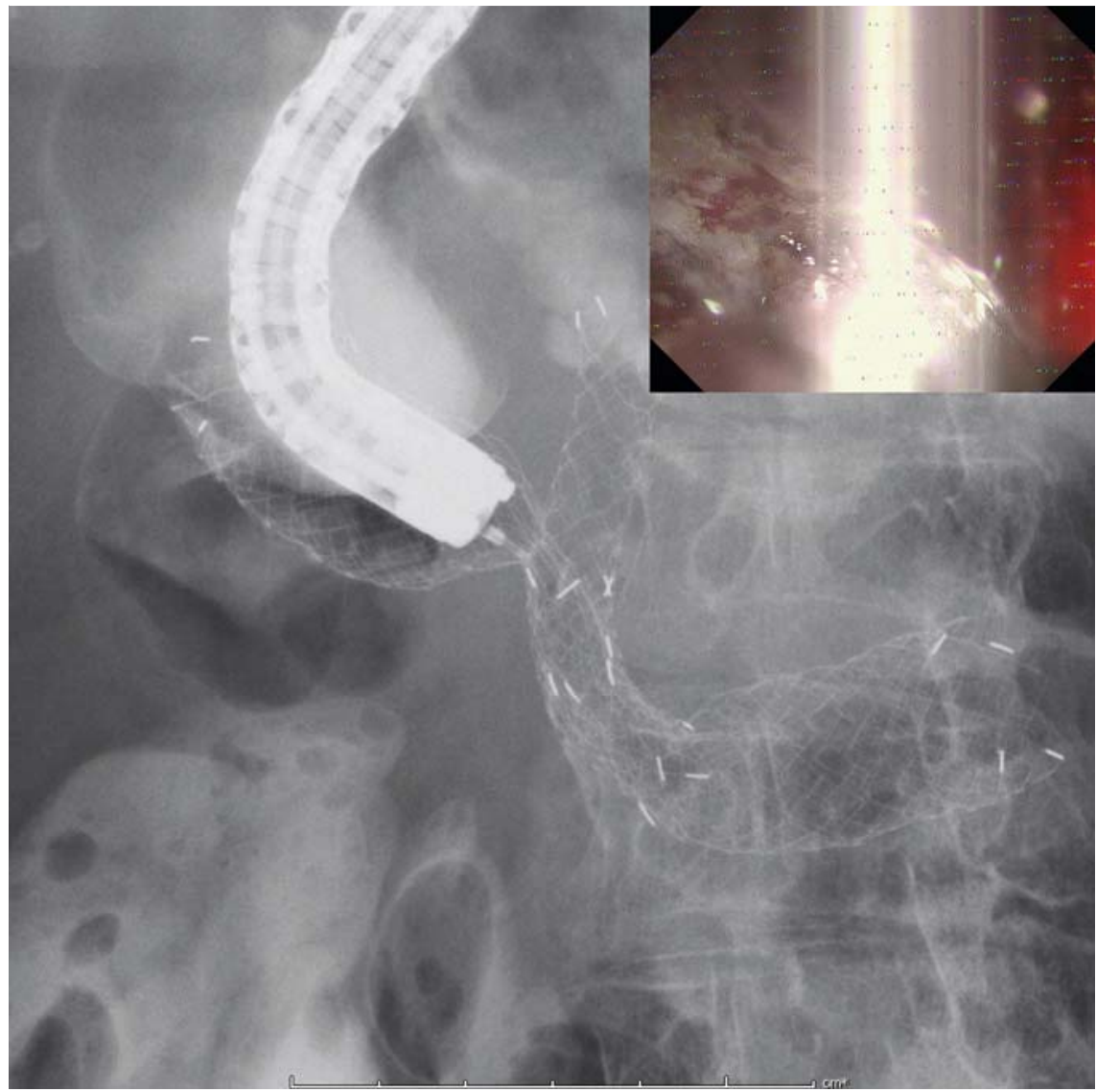

- Fig. 4 The mesh was broken in the duodenal and bile duct stents using a fine-gauge electrocautery dilator.

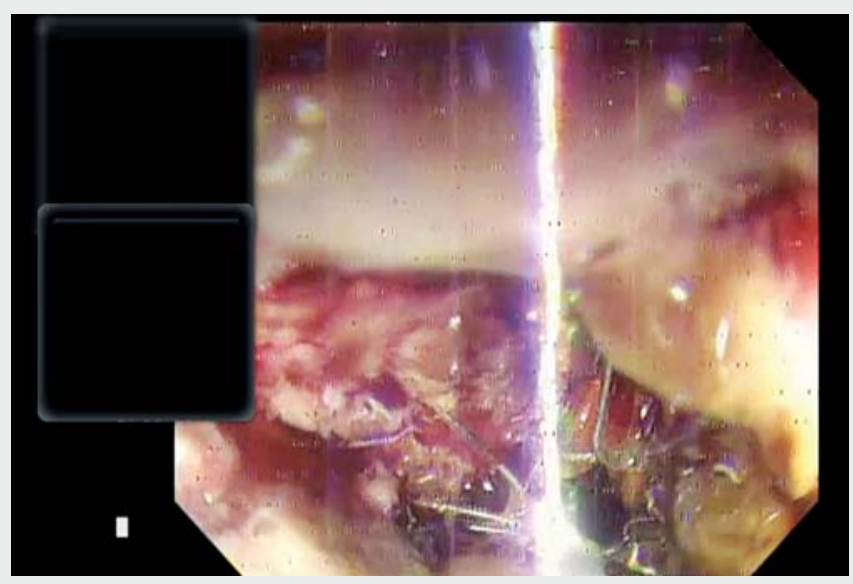

Video 1 The duodenal stent became distorted because it was placed through the mesh of the biliary stent. The mesh was broken using a fine-gauge electrocautery dilator and the duodenal stent was recanalized.
Endoscopy_UCTN_Code_CPL_1AH_2AD

Competing interests

The authors declare that they have no conflict of interest.

The authors

\section{Atsushi Okuda , Takeshi Ogura, Masanori} Yamada, Saori Ueno, Kazuhide Higuchi

Second Department of Internal Medicine, Osaka Medical College, Osaka, Japan

\section{Corresponding author}

\section{Takeshi Ogura, MD}

Second Department of Internal Medicine, Osaka Medical College, 2-7 Daigakuchou, Takatsukishi, Osaka 569-8686, Japan oguratakeshi0411@yahoo.co.jp

\section{References}

[1] Fisher AV, Hanlon B, Fernandes-Taylor S et al. Natural history and cost analysis of surgical bypass versus endoscopic stenting for the palliative management of malignant gastric outlet obstruction. HPB (Oxford) 2020; 4: 529-536

[2] Tringali A, Giannetti A, Adler DG et al. Endoscopic management of gastric outlet obstruction disease. Ann Gastroenterol 2019; 32: $330-337$

[3] Hamada T, Hakuta R, Takahara N et al. Covered versus uncovered metal stents for malignant gastric outlet obstruction: systematic review and meta-analysis. Dig Endosc 2017; 29: 259-271

[4] Miyabe K, Hayashi K, Nakazawa T et al. Safety and benefits of self-expandable metallic stents with chemotherapy for malignant gastric outlet obstruction. Dig Endosc 2015; 27: $572-581$

[5] Ogura T, Nakai Y, Iwashita T et al. Novel fine gauge electrocautery dilator for endoscopic ultrasound-guided biliary drainage: experimental and clinical evaluation study (with video). Endosc Int Open 2019; 7: E1652E1657 


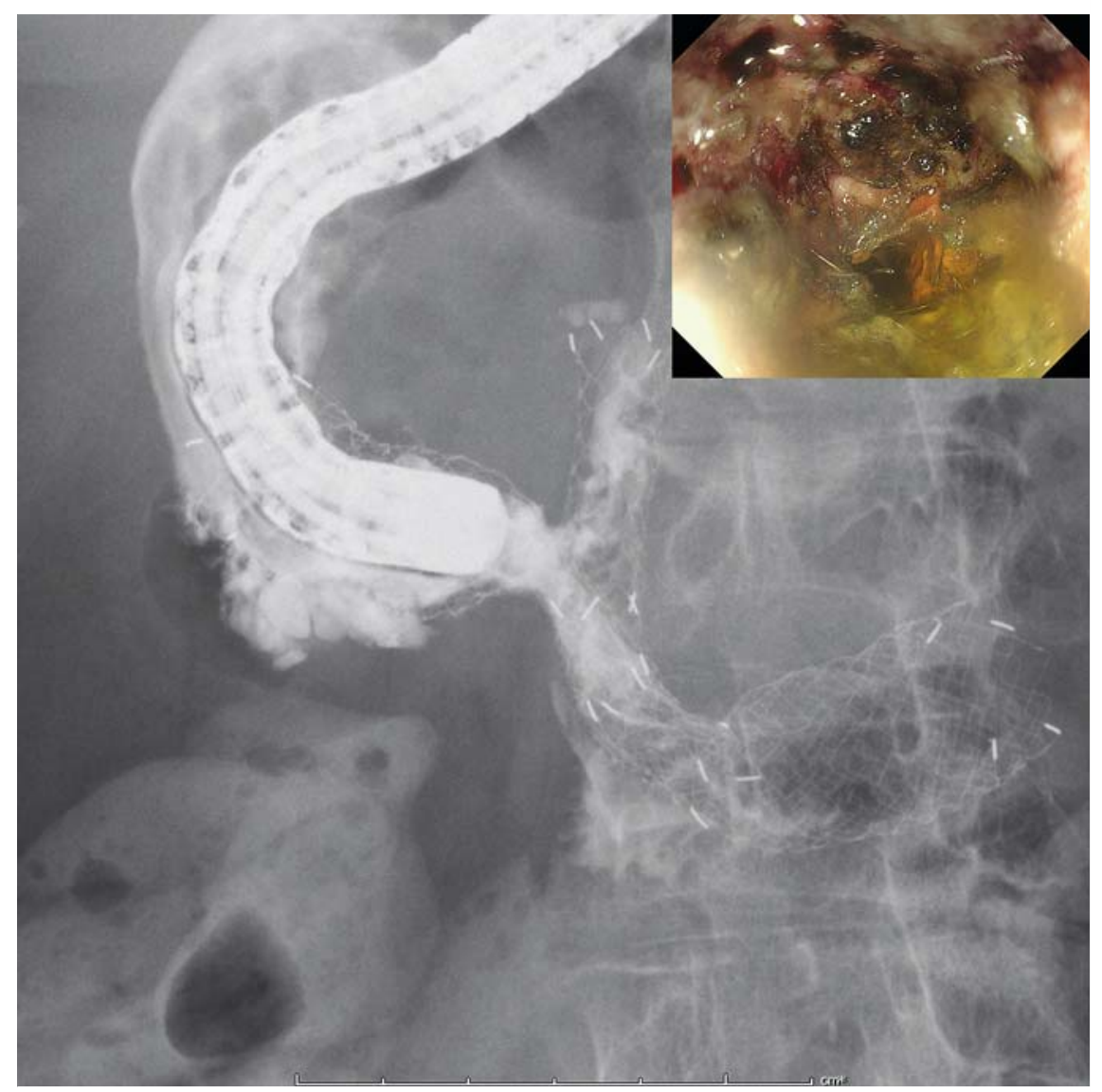

Bibliography

Endoscopy 2022; 54: E146-E148

DOI 10.1055/a-1408-0860

ISSN 0013-726X

published online 20.4.2021

(c) 2021. Thieme. All rights reserved.

Georg Thieme Verlag KG, Rüdigerstraße 14,

70469 Stuttgart, Germany

\section{ENDOSCOPY E-VIDEOS \\ https://eref.thieme.de/e-videos}

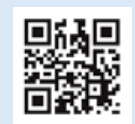

Endoscopy E-Videos is an open access online section, reporting on interesting cases and new techniques in gastroenterological endoscopy. All papers include a high quality video and all contributions are freely accessible online. Processing charges apply (currently EUR 375), discounts and wavers acc. to HINARI are available.

This section has its own submission website at

https://mc.manuscriptcentral.com/e-videos

- Fig. 5 The duodenal stent was recanalized. 